\title{
Classes of graphs with $e$-positive chromatic symmetric function
}

\author{
Angèle M. Foley* Chính T. Hoàng Owen D. Merkel ${ }^{\dagger}$ \\ Department of Physics and Computer Science \\ Wilfrid Laurier University \\ Waterloo, Ontario, Canada. \\ \{ahamel, choang\}@wlu.ca owen.merkel@uwaterloo.ca
}

Submitted: Sep 28, 2018; Accepted: Aug 7, 2019; Published: Sep 13, 2019

(C) The authors. Released under the CC BY-ND license (International 4.0).

\begin{abstract}
In the mid-1990s, Stanley and Stembridge conjectured that the chromatic symmetric functions of claw-free co-comparability (also called incomparability) graphs were $e$-positive. The quest for the proof of this conjecture has led to an examination of other, related graph classes. In 2013 Guay-Paquet proved that if unit interval graphs are $e$-positive, that implies claw-free incomparability graphs are as well. Inspired by this approach, we consider a related case and prove that unit interval graphs whose complement is also a unit interval graph are $e$-positive. We introduce the concept of strongly $e$-positive to denote a graph whose induced subgraphs are all $e$-positive, and conjecture that a graph is strongly $e$-positive if and only if it is (claw, net)-free.
\end{abstract}

Mathematics Subject Classifications: 05E05, 05C15

\section{Introduction}

A 1995 paper of Stanley [19] introduced the chromatic symmetric functions and proved a host of properties about them. A key element of this foundational paper was a conjecture due to Stanley and Stembridge (originally stated in other terms in [21]) that the chromatic symmetric functions of claw-free co-comparability (also called incomparability) graphs had the property known as e-positivity (defined in Section 2). As of this writing, this conjecture remains unproved, and work on it and on related results has fueled research

\footnotetext{
*Formerly Angèle M. Hamel.

†Current address: David R. Cheriton School of Computer Science, University of Waterloo, Waterloo, Ontario, Canada.
} 
in the area for over 20 years. A fundamental contribution to this endeavour was GuayPaquet's result that if Stanley and Stembridge's conjecture holds for unit interval graphs, then it holds for claw-free co-comparability graphs [8]. This result has put a spotlight on unit interval graphs. In related work, Shareshian and Wachs [17] generalized the conjecture of Stanley and Stembridge, introducing an extra $t$ parameter, and also conjectured a relation between the chromatic symmetric function and the natural representation of the symmetric group on the cohomology of an algebraic variety. In this setting, Harada and Precup [9] proved a special case of the Shareshian and Wachs generalization. Cho and Huh [1] and Harada and Precup [9] have proved $e$-positivity for several subclasses of unit interval graphs. The time is ripe for further investigations of subclasses and superclasses of unit interval graphs.

Graphs and their complements are natural pairs to study. Recall that the complement of a graph $G=(V, E)$ is the graph with vertices $V$ and edges between any two vertices $u, v \in V$ precisely when there is no edge between $u$ and $v$ in $G$. We use notations $\bar{G}$ or $c o-G$ to refer to the complement of $G$. The (claw, co-claw)-free graphs hold particular interest. Two of the authors investigated them in [10], concluding they were not all $e$ positive. Here we revisit this result, showing that the particular graph called the net is the only exception. This result follows by careful consideration of the graph structure, and subsequent decomposition into constituent graphs. From this analysis, along with a number of powerful graph theory results, we derive a series of results, culminating in a theorem that states that if a graph $G$ and its complement are both unit interval graphs, then $G$ is $e$-positive. We present two proofs of this, one following along the lines we set out here, and one due to Timothy Y. Chow, using classical results to show that graphs that satisfy these conditions are $e$-positive.

The graph class universe we are working in is captured by Figure 1. The class of claw-free co-comparability graphs targeted by Stanley and Stembridge wholly contains the subclass of unit interval graphs. If we look at the larger picture we see that the superclass of claw-free, AT-free graphs (see definition of AT-free in Section 2) consists of co-triangle-free graphs (known to be $e$-positive [18], restated in Theorem 4) and clawfree co-comparability graphs. Thus proving the Stanley and Stembridge conjecture would prove all claw-free, AT-free graphs were $e$-positive.

Even farther beyond this is the class of (claw, net)-free graphs. The net (see Figure 2 ) is significant as this is the example originally given by Stanley [19] of a claw-free, non-e-positive, graph to show claw-free alone is not a property sufficient to guarantee $e$-positivity. We focus particularly on (claw, net)-free graphs (note that for $n=4$ there is one non-e-positive graph (namely, the claw, $K_{1,3}$ ), for $n=5$ there are 4 non-e-positive connected graphs (namely $K_{1,4}$, dart, cricket $=K_{1,4}+e$, co- $\left\{K_{3} \cup 2 K_{1}\right\}$ ), for $n=6$, there are 44 non-e-positive connected graphs, and for $n=7$ there are 374 non-e-positive connected graphs). To our knowledge, this paper is the first exploration of the (claw, net)free $e$-positivity question. We conjecture these graphs are $e$-positive. We have verified our conjecture for graphs up to 9 vertices. We also introduce the term strongly e-positive to denote graphs whose induced subgraphs are also $e$-positive, and we conjecture a graph is strongly $e$-positive if and only if it is (claw, net)-free. 


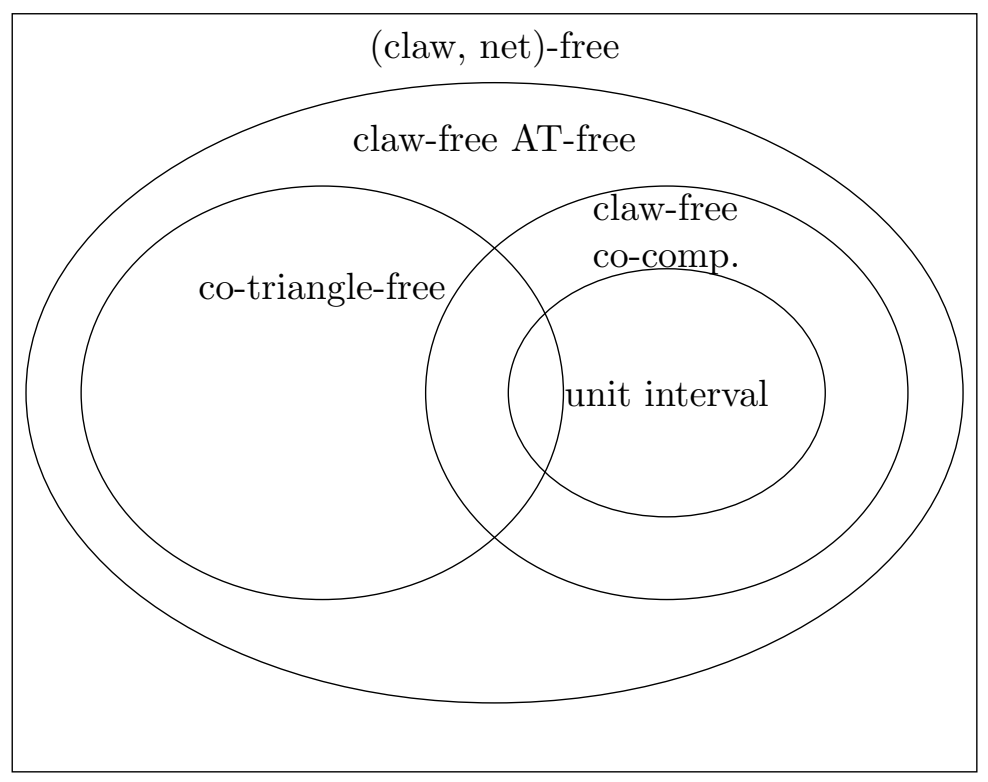

Figure 1: Classes of (claw, net)-free graphs. If the graphs are connected, then a claw-free AT-free graph is either a co-triangle-free graph or a claw-free co-comparability graph (or both).

The paper is structured as follows. Section 2 covers background and notation from both graph theory and symmetric function theory. It also summarizes much of what is already known about which graphs are $e$-positive. Section 3 proves our result on the $e$-positivity of unit interval graphs whose complement is also a unit interval graph. Along the way we consider the e-positivity question for (claw, co-claw)-free graphs. Section 4 contains our conjectures about strongly e-positive graphs and about (claw, net)-free graphs.

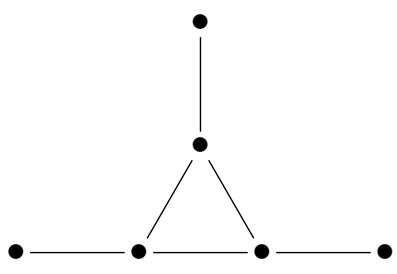

(a) The net

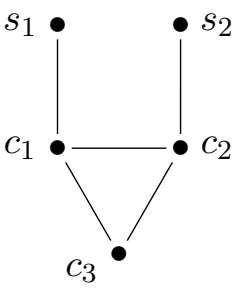

(b) The bull

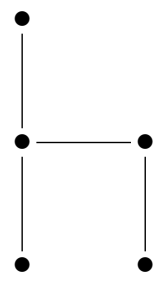

(c) The chair

Figure 2: The net, bull and chair graphs. 


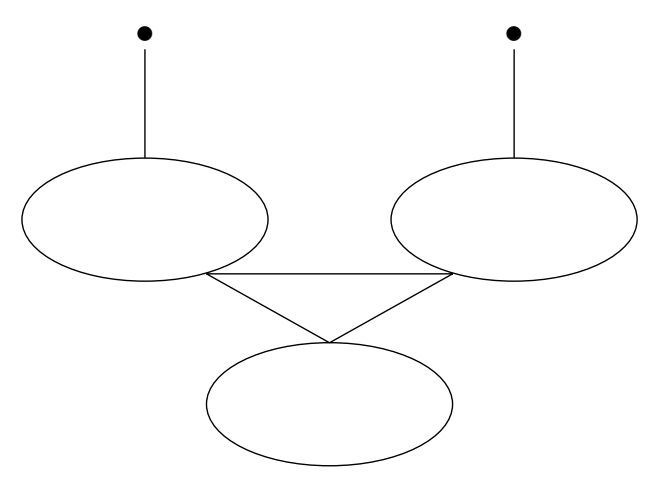

Figure 3: The generalized bull

\section{Background and Notation}

We begin by defining both graph theory and symmetric function terms and notation. Let $G=(V, E)$ be a finite, simple, undirected graph with vertex set $V$ and edge set $E$. We assume all graphs are connected, an assumption necessary because of Lemma 2. For vertices $u, v \in V$, define $d(u, v)$ to be the length of the shortest path between $u$ and $v$. For a vertex $v \in V$, the open neighbourhood of $v$ is defined by $N(v)=\{u \in V: u v \in E\}$. For $U \subseteq V$, let $[U]$ denote the induced subgraph of $G$ induced by $U$. For a set $H$ of graphs, define $H$-free to be the class of graphs that do not contain any graph in $H$ as an induced subgraph.

Let $P_{k}$ be the chordless path on $k$ vertices and $C_{k}$ be the chordless cycle on $k$ vertices. The complete graph (or clique) $K_{n}$ is the graph on $n$ vertices such that there is an edge between all pairs of vertices. A $K$-chain is a graph that is a sequence of complete graphs attached to one another sequentially at a single vertex, i.e. a vertex can belong to at most two maximal cliques. The graph $K_{3}$ is called the triangle, and its complement $3 K_{1}$, is called the co-triangle. The bull graph is the graph on 5 vertices and 5 edges arranged as a triangle with two pendant edges. See Figure 2. The generalized bull graphs are the family of graphs that can be constructed from the bull graph where each vertex in the triangle of the bull is substituted by a clique (nonempty), i.e. every vertex is replaced by a clique and there are all edges between any two of these cliques. See Figure 3.

A stable set is a set $S$ of vertices of a graph such that there are no edges between any of the vertices in $S$, e.g. a co-triangle is a stable set of size 3. Let $\alpha(G)$ denote the size of the largest stable set in $G$. An astroidal triple $(A T)$ in a graph $G$ is a stable set of three vertices in $G$ such that for any pair of vertices in the set, there is a path between them that does not intersect the neighbourhood of the third. A graph is called AT-free exactly when it does not contain an astroidal triple.

A coloring of a graph $G$ is a function $\kappa$ from $V$ to the positive integers $\mathbb{Z}^{+}: \kappa: V \rightarrow \mathbb{Z}^{+}$. A coloring $\kappa$ is proper if $\kappa(u) \neq \kappa(v)$ whenever vertex $u$ is adjacent to vertex $v$. Chromatic symmetric functions were defined by Stanley [19] as a generalization of the chromatic polynomial. Indeed, if we set $x_{1}=x_{2}=x_{3}=\ldots=x_{n}=1$ and any remaining variables 
to zero, this expression reduces to the chromatic polynomial for a graph.

Definition 1. For a graph $G$ with vertex set $V=\left\{v_{1}, v_{2}, \ldots, v_{N}\right\}$ and edge set $E$, the chromatic symmetric function is defined to be

$$
X_{G}=\sum_{\kappa} x_{\kappa\left(v_{1}\right)} x_{\kappa\left(v_{2}\right)} \cdots x_{\kappa\left(v_{N}\right)}
$$

where the sum is over all proper colorings $\kappa$ of $G$.

A function is symmetric if a permutation of the variables does not change the function. In precise terms, using the wording of Stanley [18, p286], "it is a formal power series $\sum_{\alpha} c_{\alpha} x^{\alpha}$ where (a) $\alpha$ ranges over all weak compositions $\alpha=\left(\alpha_{1}, \alpha_{2}, \ldots\right)$ of $n$ (of infinite length), (b) $c_{\alpha} \in R$, (c) $x^{\alpha}$ stands for the monomial $x_{1}^{\alpha_{1}} x_{2}^{\alpha_{2}} \ldots$, and (d) $f\left(x_{w(1)}, x_{w(2)}, \ldots\right)=f\left(x_{1}, x_{2}, \ldots\right)$ for every permutation $w$ of the positive integers" (note that $R$ is a commutative ring with identity). Full background details can be found in Macdonald [16] or Stanley [18]. It is well-known that certain sets of symmetric functions act as bases for the algebra of symmetric functions. One such set is the set of elementary symmetric functions. The ith elementary symmetric function, $e_{i}(x)$, is defined as

$$
e_{i}(x)=\sum_{j_{1}<j_{2}<\cdots<j_{i}} x_{j_{1}} \cdots x_{j_{i}}
$$

We can extend this definition using partitions. A partition $\lambda=\left(\lambda_{1}, \lambda_{2}, \ldots, \lambda_{\ell}\right)$ of a positive integer $n$ is a nonincreasing sequence of positive integers: $\lambda_{1} \geqslant \lambda_{2} \geqslant \ldots \geqslant \lambda_{\ell}$, where $\lambda_{i}$ is called the $i$ th part of $\lambda, 1 \leqslant i \leqslant \ell$. The transpose, $\lambda^{\prime}$, of $\lambda$, is defined by its parts: $\lambda_{i}^{\prime}=\left|\left\{j: \lambda_{j} \geqslant i\right\}\right|$. The elementary symmetric function, $e_{\lambda}(x)$, is defined as $e_{\lambda}(x)=e_{\lambda_{1}} e_{\lambda_{2}} \ldots e_{\lambda_{\ell}}$. Note that we will use the notation $e_{i}$ for $e_{i}(x)$ and $e_{\lambda}$ for $e_{\lambda}(x)$.

If a given symmetric function can be written as a nonnegative linear combination of elementary symmetric functions we say the symmetric function is e-positive. By abuse of notation we say a graph is $e$-positive if its chromatic symmetric function is $e$-positive. Furthermore, we say that a class of graphs is $e$-positive if every graph in the class is $e$-positive.

Note that the property of a graph being $e$-positive is not hereditary. That is, if a graph is $e$-positive, all of its induced subgraphs are not necessarily $e$-positive. For example, the chair (or fork) graph (see Figure 2) is $e$-positive with chromatic symmetric function $X_{F}=e_{2,2,1}+2 e_{3,1,1}+e_{3,2}+7 e_{4,1}+5 e_{5}$, but contains an induced claw $K_{1,3}$ which is not $e$-positive, as $X_{K_{1,3}}=e_{4}+5 e_{3,1}-2 e_{2,2}+e_{2,1,1}$. In Section 4 we consider graphs whose induced subgraphs are all $e$-positive and dub these graphs strongly e-positive.

The following lemmas from Stanley [19] is useful in constructing new classes of graphs:

Lemma 2 ([19], Proposition 2.3). If a graph $G$ is a disjoint union of subgraphs $G_{1} \cup G_{2}$, then $X_{G}=X_{G_{1}} X_{G_{2}}$.

Lemma 3 ([19], Corollary 3.6). If the vertices can be partitioned into disjoint cliques, then the graph is e-positive. 


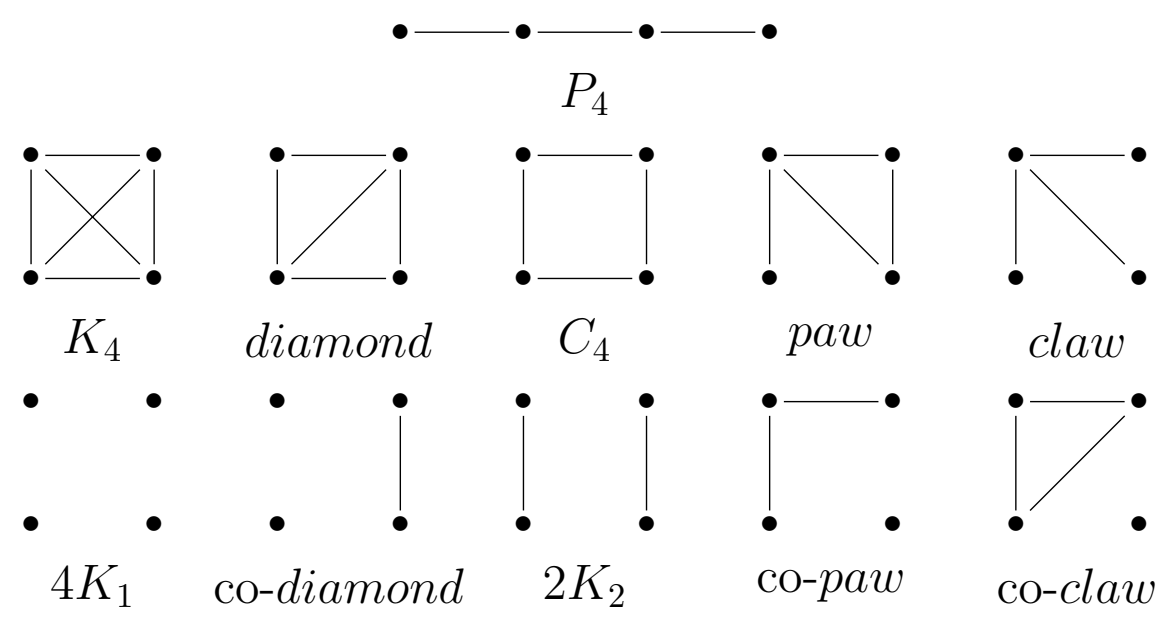

Figure 4: All four-vertex graphs.

\begin{tabular}{||c|c|c||}
\hline \hline Set $H$ & Positivity & Reference \\
\hline \hline$P_{3}$ & $e$-positive & Theorem 5 \\
\hline $3 K_{1}$ & $e$-positive & {$[18]$} \\
\hline claw, $K_{3}$ & $e$-positive & Theorem 5 \\
\hline claw, co- $P_{3}$ & $e$-positive & Theorem 5 \\
\hline \hline
\end{tabular}

Figure 5: Table of $e$-positivity for $H$-free graphs where $H$ contains a three-vertex graph.

\begin{tabular}{||c|c|c||}
\hline \hline Set $H$ & Positivity & Reference \\
\hline \hline claw, $P_{4}$ & $e$-positive & {$[22]$} \\
\hline claw, paw & $e$-positive & {$[10]$} \\
\hline claw, co-paw & $e$-positive & {$[10]$} \\
\hline claw, co-claw (excluding the net) & $e$-positive & Corollary 17 \\
\hline claw, co-diamond & conjectured $e$-positive & Conjecture 22 \\
\hline claw, diamond & not necessarily $e$-positive & {$[10]$} \\
\hline claw, $K_{4}$ & not necessarily $e$-positive & {$[10]$} \\
\hline claw, $4 K_{1}$ & not necessarily $e$-positive & {$[10]$} \\
\hline claw, $C_{4}$ & not necessarily $e$-positive & {$[10]$} \\
\hline claw, $2 K_{2}$ & not necessarily $e$-positive & {$[10]$} \\
\hline \hline
\end{tabular}

Figure 6: Table of $e$-positivity for $H$-free graphs where $H$ contains four-vertex graphs. Note that the exceptional case, the net, is not $e$-positive. 
The following theorem summarizes the $e$-positive status of a number of graph classes:

Theorem 4. The following graph classes are known to be e-positive (proofs in the individual references given):
1. $P_{k}[19]$
2. $C_{k}[19]$
3. $K_{n}$ [2]; implicit in [19]
4. co-triangle-free [18, p514]
5. K-chains [7]
6. generalized bull [1]
7. (claw, $P_{4}$-free) [22]
8. (claw, paw)-free [10]
9. (claw, co-paw)-free [10]

Theorem 5. The following graph classes are e-positive:
1. $P_{3}$-free
2. (claw, triangle)-free
3. (claw, co- $\left.P_{3}\right)$-free

Proof. We have the following arguments:

Item 1: If $G$ is $P_{3}$-free then the components of $G$ are cliques. By [2], restated in Theorem 4 , and Lemma $2, G$ is $e$-positive.

Item 2: If $G$ is (claw, triangle)-free, then each component of $G$ is a chordless path or cycle. Then together with [19], restated in Theorem 4, and Lemma 2, $G$ is e-positive.

Item 3: The class of (claw, co- $P_{3}$ )-free graphs is a subclass of (claw, co-paw)-free graphs which was shown to be $e$-positive in [10].

Finally, this is Stanley and Stembridge's celebrated conjecture. They expressed it in terms of incomparability graphs of $(3+1)$-free posets.

Conjecture 6 ([21]). A claw-free, co-comparability graph is $e$-positive. 
In [10] classes of (claw, $H$ )-free graphs were analyzed, where $H$ is a graph on 4 vertices. However several classes of graphs, including (claw, diamond)-free graphs and (claw, coclaw)-free graphs are not necessarily $e$-positive, as was demonstrated using a counterexample. The counter-example that is used is a six-vertex graph called the net (Figure 2 ). Here we extend the results of [10] to remark that there are infinite families of non-epositive graphs:

Theorem 7. There are infinitely many (claw, diamond)-free graphs, (claw, $C_{4}$ )-free graphs, (claw, $K_{4}$ )-free graphs, and (claw, $2 K_{2}$ )-free graphs that are not e-positive.

Proof. The family of triangle tower graphs, described in [4] are not $e$-positive, but are (claw, diamond, $C_{4}, K_{4}$ )-free. The family of generalized nets, described in [5] are not $e$-positive, but are (claw, $2 K_{2}$ )-free.

Returning to Stanley's singular counter-example - the net-we focus on this special graph. In the next section we will show it is the only (claw, co-claw)-free graph that is not $e$-positive. We also note that the net contains an asteroidal triple and this causes us to turn our focus to AT-free graphs. In particular, we note this significant result of Kloks, Kratsch, and Müller which shows that the claw-free, co-comparability graphs are one half of the set of (claw, AT)-free graphs.

Theorem 8 ([14]). A connected graph $G$ is claw-free and AT-free if and only if at least one of the following holds:

1. G is a claw-free co-comparability graph.

2. $G$ is co-triangle-free.

Together with a result from [18] (restated in Theorem 4), Conjecture 6 would imply that the class of claw-free AT-free graphs is $e$-positive.

\section{Unit interval graphs}

An interval graph $G$ is a graph whose vertices can be represented by intervals on a straight line where two vertices in $G$ are adjacent if and only if their corresponding intervals intersect. A unit interval graph is an interval graph whose intervals are given by unit lengths. It has been shown in [15] that interval graphs are exactly the class of chordal $A T$-free graphs, where a graph is a chordal graph if each of its cycles of length at least four has a chord, i.e. an edge that is not part of the cycle but connects two vertices of the cycle. At the same time, unit interval graphs have been shown to be exactly the class of clawfree interval graphs [6]. Guay-Paquet [8] has proved that Conjecture 6 can be reduced to the statement that the chromatic symmetric function of unit interval graphs are $e$ positive. So proving certain classes of unit interval graphs are $e$-positive will support this conjecture. This gives the motivation to study $H$-free unit interval graphs or equivalently (claw, $H$ )-free $A T$-free chordal graphs. 


\begin{tabular}{||c|c|c||}
\hline \hline Graph $H$ & Positivity & Reference \\
\hline \hline$P_{4}$ & $e$-positive & {$[22]$} \\
\hline paw & $e$-positive & {$[10]$} \\
\hline co-paw & $e$-positive & {$[10]$} \\
\hline co-claw & $e$-positive & Theorem 18 \\
\hline diamond & $e$-positive & Theorem 10 \\
\hline co-diamond & unknown & unknown \\
\hline$K_{4}$ & unknown & unknown \\
\hline $4 K_{1}$ & unknown & unknown \\
\hline $2 K_{2}$ & unknown & unknown \\
\hline \hline
\end{tabular}

Figure 7: Table of $e$-positivity results for $H$-free unit interval graphs where $H$ is a fourvertex graph.

We investigate two different angles on the $e$-positivity question on unit interval graphs: 1) unit interval graphs that are $H$-free for $H$ a four vertex graph; and, 2) co-claw-free unit interval graphs.

\section{1 $\quad H$-free unit interval graphs}

The table in Figure 7 summarizes what is known about $H$-free unit interval graphs, where $H$ is a four vertex graph. This lemma from Hempel and Kratsch is required in what follows:

Lemma 9 ([11]). Let $G=(V, E)$ be a claw-free, AT-free graph. Let $N_{0}=\{w\}, N_{1}=N(w)$, $N_{2}, \ldots, N_{i}=\{x \in V \mid d(x, w)=i\}$ for $i \geqslant 2$. Then the following holds:

1. $N_{i}$ is a clique for all $i=0,2,3, \ldots$

2. $\alpha\left(\left[N_{1}\right]\right) \leqslant 2$.

Theorem 10. If $G$ is a diamond-free unit interval graph, then $G$ is e-positive.

Proof. Let $G=(V, E)$ be a diamond-free unit interval graph. From Lemma 9, fix $w \in V$ and define $N_{0}=\{w\}, N_{1}=N(w), N_{2}, \ldots, N_{i}=\{x \in V \mid d(x, w)=i\}$ for $i \geqslant 2$. By Lemma $9, N_{i}$ is a clique for all $i \neq 1$. Since $G$ is diamond-free, $\left[N_{1}\right]$ must be $P_{3}$-free. Then since $\alpha\left(\left[N_{1}\right]\right) \leqslant 2$, either $\left[N_{1}\right]$ is a complete graph or the disjoint union of two complete graphs.

Case 1. Suppose $\left[N_{1}\right]$ is a complete graph.

Every vertex in $N_{2}$ must have exactly one neighbour in $N_{1}$. If $y \in N_{2}$ has two neighbours $x_{1}, x_{2} \in N_{1}$ then $\left\{w, x_{1}, x_{2}, y\right\}$ induces a diamond. Then every vertex in $N_{2}$ must be adjacent to the same vertex in $N_{1}$ say $x$, or $G$ will contain an induced $C_{4}$, which contradicts $G$ being chordal. For $i \geqslant 0$, this arguement can be continuely applied to $N_{i+2}$ since there is a vertex in $N_{i}$ that every vertex in $N_{i+1}$ is adjacent to. Therefore $G$ is a $K$-chain and by [7], restated in Theorem $4, G$ is e-positive. 


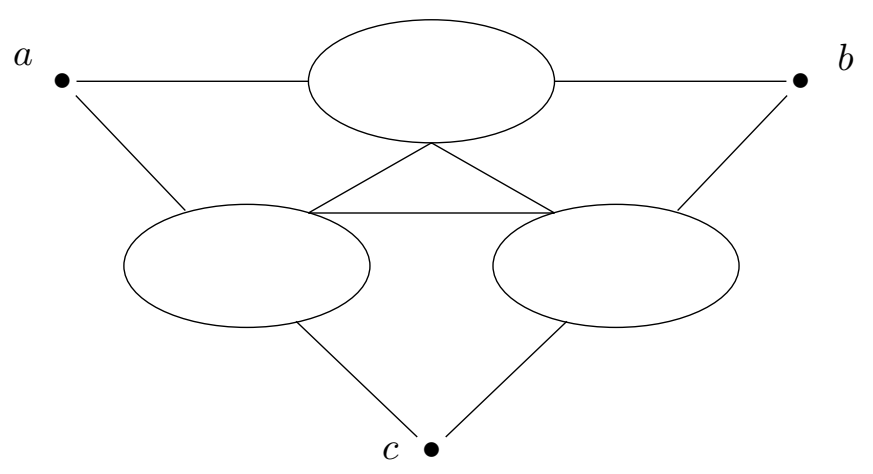

Figure 8: A generalized pyramid

Case 2. Suppose $\left[N_{1}\right]$ is the disjoint union of two complete graphs.

Note that no vertex in $N_{2}$ can have more than one neighbour in $N_{1}$. If a vertex in $N_{2}$ has two neighbours in the same component of $\left[N_{1}\right]$, then $G$ will contain an induced diamond. If a vertex in $N_{2}$ has a neighbour in each component of $\left[N_{1}\right]$, then $G$ will contain an induced $C_{4}$. Thus every vertex in $N_{2}$ has exactly one neighbour in $N_{1}$. Every vertex in $N_{2}$ has the same neighbour in $N_{1}$ or $G$ will contain an induced $C_{4}$ or $C_{5}$. Then one component of $\left[N_{1}\right]$ has no neighbours in $N_{2}$. For $i \geqslant 0$, a similar argument can be continually applied to $N_{i+2}$ since there is a vertex in $N_{i}$ that every vertex in $N_{i+1}$ is adjacent to. Therefore $G$ is a $K$-chain and by [7], restated in Theorem $4, G$ is $e$-positive.

We also remark that it can be shown that a graph is a $K$-chain graph if and only if it is diamond-free unit interval graph.

In the next theorem we will make use of the following term: $G$ is a generalized pyramid if every oval is a clique and there are all edges between any two ovals if there was an edge between them in the original graph (see Figure 8).

Theorem 11. If $G$ is a ( $2 K_{2}$, co-diamond)-free unit interval graph, then $G$ is e-positive.

Proof. From [10], it was determined that (claw, co-diamond, $2 K_{2}$ )-free graphs that are not known to be $e$-positive are the generalized pyramid graphs. Note that if all 3 ovals of $G$ are non-empty, then $G$ contains an astroidal triple $\{a, b, c\}$. Since unit interval graphs are $A T$-free, it must be the case that one or two ovals of $G$ are empty. In either case, $G$ is a generalized bull graph, and from [1], restated in Theorem 4, $G$ is $e$-positive.

As a side issue we can consider the families of $2 K_{2}$-free unit interval graphs that are not known to be e-positive. Let $G=(V, E)$ be a $2 K_{2}$-free unit interval graph. We can assume $G$ is connected since if not $G$ has at most one component that is not an isolated vertex. From Lemma 9, fix $w \in V$ and define $N_{0}=\{w\}, N_{1}=N(w)$, $N_{2}, \ldots, N_{i}=\{x \in V \mid d(x, w)=i\}$ for $i \geqslant 2$. Since $G$ is $2 K_{2}$-free, $N_{i}=\varnothing$ for all $i \geqslant 4$. Then by Lemma $9, N_{i}$ is a clique for $i=0,2,3$ and $\alpha\left(\left[N_{1}\right]\right) \leqslant 2$. If $N_{3}$ has an edge $\left\{z_{1} z_{2}\right\}$ then for any vertex $x \in N_{1},\left\{w, x, z_{1}, z_{2}\right\}$ induces a $2 K_{2}$ in $G$. Then either $N_{3}$ is empty or $N_{3}$ has a single vertex. 
Case 1. Suppose $\left[N_{1}\right]$ is not connected.

Then $\left[N_{1}\right]$ has two components, say $N_{1,0}$ and $N_{1,1}$, otherwise G contains an induced claw. At least one component of $\left[N_{1}\right]$ is a single vertex, say $N_{1,1}$, and the other is a clique. If $N_{2}$ is empty, then $G$ can be partitioned into 2 cliques, $N_{0} \cup N_{1,0}$ and $N_{1,1}$, and by [19], restated in Lemma 3, $G$ is e-positive. Now suppose $y_{1} \in N_{2}$. Then vertices in $N_{2}$ can only have neighbours in one component of $\left[N_{1}\right]$ or $G$ will have a chordless cycle on at least 4 vertices. Then if $N_{3} \neq \varnothing$ or $\left[N_{2}\right]$ has an edge, this edge together with the edge formed by $w$ and a vertex from the component of $\left[N_{1}\right]$ with no neighbours in $N_{2}$, will induce a $2 K_{2}$ in $G$. Then $N_{2}$ has exactly one vertex and $N_{3}$ is empty. In this case $G$ is a generalized bull, and by [1], restated in Theorem $4, G$ is $e$-positive.

Case 2. Suppose $\left[N_{1}\right]$ is connected.

Suppose $N_{3}$ has a single vertex, $z$. Any vertex $y_{1} \in N_{2}$ that is adjacent to $z$ must be adjacent to every vertex in $N_{1}$. If $y_{1}$ is not adjacent to $x \in N_{1}$, then $\left\{w, x, y_{1}, z\right\}$ induces a $2 K_{2}$ in $G$. Also any vertex $y_{2} \in N_{2}$ that is not adjacent to $z$ must be adjacent to every vertex in $N_{1}$. If $y_{2}$ is not adjacent to $x \in N_{1}$ then $\left\{y_{1}, y_{2}, z, x\right\}$ induces a claw in $G$. It must also be the case that $N_{1}$ is a clique. If $x_{1}, x_{2} \in N_{1}$ are not adjacent then $\left\{x_{1}, x_{2}, y_{1}, z\right\}$ induces a claw in $G$. Then $G$ is a generalized bull and by [1], restated in Theorem 4, $G$ is $e$-positive.

Now suppose $N_{3}$ is empty. Then if $N_{1}$ is a clique, $G$ can be partitioned into two cliques, $N_{0} \cup N_{1}$ and $N_{2}$, and by [19], restated in Lemma 3, $G$ is e-positive. Then $N_{1}$ must contain an induced $P_{3}$.

The family of $2 K_{2}$-free unit interval graphs that are not known to be $e$-positive have $\left[N_{1}\right]$ connected, $\left[N_{1}\right]$ contains an induced $P_{3}, \alpha\left(\left[N_{1}\right]\right)=2, N_{2} \neq \varnothing$, and all $N_{i}=\varnothing$ for $i \geqslant 3$.

\subsection{The structure of (claw, co-claw)-free graphs}

As a preliminary to considering co-claw, unit interval graphs, we investigate (claw, coclaw)-free graphs. First observe that the net is a (claw, co-claw)-free graph that is not $e$-positive. Here we will show this is the only graph in this class that is not $e$-positive. Theorems 12 and 13 below are implicitly implied by the proof of Theorem 3 in [13]. For the sake of completeness, we will give a proof of both theorems here. Note that the complement of the net is called the 3-sun.

Theorem 12 ([13]). Let $G$ be a (claw, co-claw)-free graph. If $G$ contains a triangle and a co-triangle that are vertex-disjoint, then $G$ contains a net or a 3-sun as an induced subgraph.

Proof. Let $G$ be a (claw, co-claw)-free graph. Suppose $G$ contains a triangle $T$ with vertices $c_{1}, c_{2}, c_{3}$ and a co-triangle $C$ with vertices $s_{1}, s_{2}, s_{3}$ such that the triangle and co-triangle are vertex-disjoint. We claim that

For a triangle $R$, every vertex in $G-R$ is adjacent to at least one vertex in $R$.

THE ELECTRONiC JOURNAL OF COMBINATORICS 26(3) (2019), \#P3.51 
If (1) failed then $R$ and a vertex of $G-R$ with no neighbors in $R$ would form a co-claw with $R$, a contradiction.

Suppose $s_{1}$ is adjacent to all vertices of the triangle $T$. Consider the triangle $\left\{s_{1}, c_{1}, c_{2}\right\}$. By (1), vertex $s_{2}$ is adjacent to $c_{1}$, or $c_{2}$. We may assume $s_{2}$ is adjacent to $c_{2}$. We have the following implications:

- the vertex $c_{2}$ is not adjacent to $s_{3}$, for otherwise $\left\{c_{2}, s_{1}, s_{2}, s_{3}\right\}$ induces a claw, a contradiction

- the vertex $s_{3}$ is adjacent to $c_{1}$, for otherwise $\left\{s_{3}, c_{1}, c_{2}, s_{1}\right\}$ induces a co-claw, a contradiction

- the vertex $s_{3}$ is adjacent to $c_{3}$, for otherwise $\left\{s_{3}, c_{2}, c_{3}, s_{1}\right\}$ induces a co-claw, a contradiction

- the vertex $s_{2}$ is not adjacent to $c_{1}$, for otherwise $\left\{c_{1}, s_{1}, s_{2}, s_{3}\right\}$ induces a claw, a contradiction

- the vertex $s_{2}$ is not adjacent to $c_{3}$, for otherwise $\left\{c_{3}, s_{1}, s_{2}, s_{3}\right\}$ induces a claw, a contradiction

- the vertex $\left\{s_{2}, c_{1}, c_{3}, s_{1}\right\}$ induces a co-claw, a contradiction.

Thus, we may assume that every $s_{i}(i=1,2,3)$ is non-adjacent to at least one $c_{j}(j=$ $1,2,3)$. Consider the vertex $s_{1}$ and suppose $s_{1}$ is adjacent to two vertices of $\left\{c_{1}, c_{2}, c_{3}\right\}$. We may assume $s_{1}$ is adjacent to $c_{1}, c_{2}$ and non-adjacent to $c_{3}$. If $c_{1}$ is adjacent to both $s_{2}, s_{3}$, then $\left\{c_{1}, s_{1}, s_{2}, s_{3}\right\}$ induces a claw, a contradiction. Thus, we may assume $c_{1}$ is not adjacent to $s_{2}$. We have the following implications:

- the vertex $s_{2}$ is adjacent to $c_{2}$, for otherwise, $\left\{s_{2}, c_{1}, c_{2}, s_{1}\right\}$ induces a co-claw

- the vertex $s_{3}$ is not adjacent to $c_{2}$, for otherwise, $\left\{c_{2}, s_{1}, s_{2}, s_{3}\right\}$ induces a claw

- the vertex $s_{3}$ is adjacent to $c_{1}$, for otherwise $\left\{s_{3}, c_{1}, c_{2}, s_{1}\right\}$ induces a co-claw

- the vertex $s_{3}$ is adjacent to $c_{3}$, for otherwise $\left\{c_{1}, s_{1}, c_{3}, s_{3}\right\}$ induces a claw

- the vertex $s_{2}$ is adjacent to $c_{3}$, for otherwise $\left\{s_{2}, c_{1}, c_{3}, s_{3}\right\}$ induces a co-claw.

Now, $T \cup C$ induces a 3 -sun in $G$. So, we may assume every vertex in $C$ is adjacent to exactly one vertex in $T$. If two vertices of $C$ are adjacent to the same vertex in $T$, then $G$ contains a claw. It is now easily to see that $T \cup C$ induces a net in $G$.

Theorem 13 ([13]). Let $G$ be a (claw, co-claw)-free graph. If $G$ contains a net or a 3-sun as an induced subgraph, then $G$ is a net, or a 3-sun. 


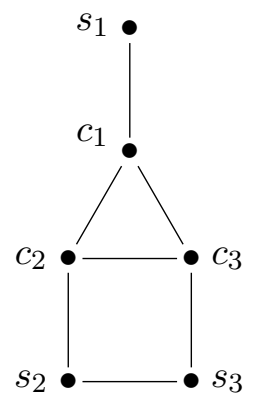

Figure 9: The antenna

Proof. Let $G$ be a (claw, co-claw)-free graph. Suppose that $G$ contains a net with vertices $c_{1}, c_{2}, c_{3}, s_{1}, s_{2}, s_{3}$ such that $C=\left\{c_{1}, c_{2}, c_{3}\right\}$ induces a triangle, $S=\left\{s_{1}, s_{2}, s_{3}\right\}$ induces a co-triangle, and $c_{i}$ is adjacent to $s_{i}$ for $i=1,2,3$. Consider a vertex $t$ not belonging to the net. Vertex $t$ cannot be adjacent to all vertices of $S$, for otherwise $S \cup\{t\}$ induces a claw. So we may assume $t$ is not adjacent to $s_{1}$. Suppose $t$ is adjacent to $c_{1}$. Since $\left\{c_{1}, s_{1}, c_{2}, t\right\}$ cannot induce a claw, $t$ must be adjacent to $c_{2}$. Similarly, since $\left\{c_{1}, s_{1}, c_{3}, t\right\}$ cannot induce a claw, $t$ must be adjacent to $c_{3}$. Now $\left\{s_{1}, t, c_{2}, c_{3}\right\}$ induces a co-claw, a contradiction.

So we know $t$ is not adjacent to $c_{1}$. Vertex $t$ must be adjacent to $c_{2}$, or $c_{3}$ (or both), for otherwise, $\left\{t, c_{1}, c_{2}, c_{3}\right\}$ induces a co-claw, a contradiction. Without loss of generality, assume $t$ is adjacent to $c_{2}$. Since $\left\{c_{2}, c_{1}, s_{2}, t\right\}$ cannot induce a claw, $t$ must be adjacent to $s_{2}$. But now $\left\{s_{1}, t, c_{2}, s_{2}\right\}$ induces a co-claw. We now can conclude that if $G$ contains a net, the $G$ is a net. By considering the complement of $G$, it follows that if $G$ contains a 3 -sun, then $G$ is a 3 -sun.

Theorem 14. Let $G$ be a (claw, co-claw)-free graph. If $G$ contains an antenna, then $G$ is an induced subgraph of the graph $F_{1}$.

Proof. Let $G$ be a (claw, co-claw)-free graph. Suppose $G$ contains an antenna $A$ with vertices $c_{1}, c_{2}, c_{3}, s_{1}, s_{2}, s_{3}$ as indicated by Figure 9 (the vertices $c_{i}$ 's form a clique, the set $\left\{s_{1}, s_{2}, s_{3}\right\}$ contains an unique edge $s_{2} s_{3}$, and $s_{i}$ is adjacent to $c_{i}$ for $i=1,2,3$.). Let $D_{1}$ be the set of vertices in $G-A$ that are adjacent to $s_{2}, s_{3}, c_{1}, s_{1}$ and no other vertices in $A$. Let $D_{2}$ be the set of vertices in $G-A$ that are adjacent to $s_{1}, c_{2}, s_{2}$ and no other vertices in $A$. Let $D_{3}$ be the set of vertices in $G-A$ that are adjacent to $s_{1}, c_{3}, s_{3}$ and no other vertices in $A$. We claim that

Every vertex in $G-A$ belong to $D_{1} \cup D_{2} \cup D_{3}$.

Let $v$ be a vertex in $G-A$. Consider the triangle $T$ with vertices $c_{1}, c_{2}, c_{3}$. Then $v$ must be adjacent to at least one vertex in $T$, for otherwise $v$ and $T$ form a co-triangle. Suppose that $v$ is adjacent to both $c_{2}$ and $c_{3}$. We have the following implications:

- the vertex $v$ is adjacent to $s_{1}$, for otherwise, $\left\{s_{1}, v, c_{2}, c_{3}\right\}$ induces a co-claw 
- the vertex $v$ is not adjacent to $s_{3}$, for otherwise, $\left\{v, s_{1}, c_{2}, s_{3}\right\}$ induces a claw

- the vertex $v$ is adjacent to $c_{1}$, for otherwise, $\left\{c_{3}, c_{1}, v, s_{3}\right\}$ induces a claw

- the vertex $\left\{s_{3}, v, c_{1}, s_{1}\right\}$ induces a co-claw, a contradiction.

So $v$ must be non-adjacent to $c_{2}$, or $c_{3}$. Suppose that $v$ is non-adjacent to both $c_{2}$ and $c_{3}$. We will show that $v$ must be in $D_{1}$. Note that $v$ is adjacent to $c_{1}$ for otherwise $\left\{v, c_{1}, c_{2}, c_{3}\right\}$ induces a co-claw. We have the following implications:

- the vertex $v$ is adjacent to $s_{1}$, for otherwise $\left\{c_{1}, s_{1}, v, c_{3}\right\}$ induces a claw

- the vertex $v$ is adjacent to $s_{3}$, for otherwise $\left\{s_{3}, v, c_{1}, s_{1}\right\}$ induces a co-claw

- the vertex $v$ is adjacent to $s_{2}$, for otherwise $\left\{s_{2}, v, c_{1}, s_{1}\right\}$ induces a co-claw

Thus, $v$ belongs to $D_{1}$. So we may assume $v$ is adjacent to exactly one vertex of $\left\{c_{2}, c_{3}\right\}$. Suppose that $v$ is adjacent to $c_{3}$ but not to $c_{2}$. We will show that $v$ belongs to $D_{3}$. We have the following implications:

- the vertex $v$ is adjacent to $s_{3}$, for otherwise, $\left\{c_{3}, c_{2}, v, s_{3}\right\}$ induces a claw

- the vertex $v$ is adjacent to $s_{1}$, for otherwise, $\left\{s_{1}, v, c_{3}, s_{3}\right\}$ induces a co-claw

- the vertex $v$ is non-adjacent to $s_{2}$, for otherwise, $\left\{v, s_{1}, c_{3}, s_{2}\right\}$ induces a claw

- the vertex $v$ is non-adjacent to $c_{1}$, for otherwise, $\left\{s_{2}, v, c_{1}, s_{1}\right\}$ induces a co-claw

Thus, $v$ belongs to $D_{3}$. By symmetry, if $v$ is adjacent to $c_{2}$ but not to $c_{3}$, then $v$ belongs to $D_{2}$. We have established (2). If some $D_{i}$ contains at least two vertices, then it is easy to see that $G$ contains a claw, or co-claw. Thus $G$ has at most 9 vertices and is an induced subgraph of $F_{1}$.

Theorem 15. Let $G$ be a (claw, co-claw)-free graph. If $G$ does not contain a net, a 3-sun, or an antenna, and contains a bull, then $G$ is an induced subgraph of the graph $F_{2}$.

Proof. Let $G$ be a (claw, co-claw)-free graph that does not contain a net, or an antenna, but contains a bull $B$. Let the vertices of the bull $B$ be $c_{1}, c_{2}, c_{3}, s_{1}, s_{2}$ as indicated by Figure 2 (the set $\left\{c_{1}, c_{2}, c_{3}\right\}$ forms a triangle, $s_{1}$ is adjacent to $c_{i}$ for $i=1,2$ ). We may assume $G$ is not the bull, for otherwise we are done. Let $X$ be the set of vertices of $G-B$ that are adjacent to $s_{1}, c_{1}, s_{2}$ and non-adjacent to $c_{2}, c_{3}$. Let $Y$ be the set of vertices of $G-B$ that are adjacent to $s_{1}, c_{2}, s_{2}$ and non-adjacent to $c_{1}, c_{3}$. We are going to show that every vertex in $G-B$ belongs to $X \cup Y$.

Consider a vertex $v \in G-B$. Suppose that $v$ is adjacent to $c_{3}$. Then $v$ is nonadjacent to at least one vertex of $\left\{s_{1}, s_{2}\right\}$, for otherwise, $\left\{v, s_{1}, s_{2}, c_{3}\right\}$ induces a claw, a contradiction. Without loss of generality, assume $v$ is non-adjacent to $s_{1}$. Then $v$ is non-adjacent to $c_{2}$, for otherwise, $\left\{s_{1}, v, c_{2}, c_{3}\right\}$ induces a co-claw. Now, $v$ is non-adjacent 


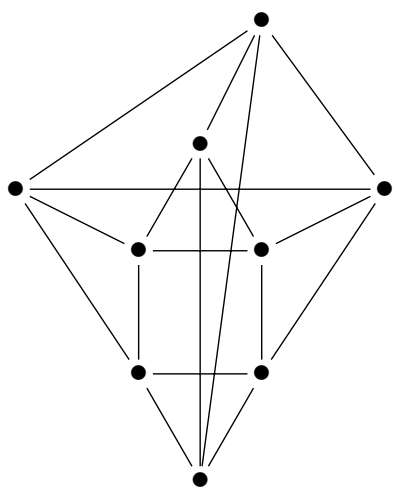

(a) $F_{1}$

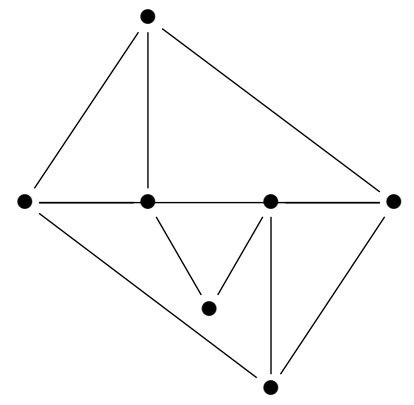

(b) $F_{2}$

Figure 10: The graphs $F_{1}$ and $F_{2}$

to $c_{1}$, for otherwise, $\left\{c_{1}, s_{1}, v, c_{2}\right\}$ induces a claw. But then the set $\left\{c_{1}, c_{2}, c_{3}, s_{1}, s_{2}, v\right\}$ induces a net or an antenna in $G$, a contradiction. So, $v$ is non-adjacent to $c_{3}$.

Consider the triangle with vertices $c_{1}, c_{2}, c_{3}$. Vertex $v$ must be adjacent to $c_{1}$, or $c_{2}$, for otherwise, the triangle and $v$ induce a co-claw. Suppose that $v$ is adjacent to $c_{1}$. Now $v$ must be adjacent to $s_{1}$, for otherwise, $\left\{c_{1}, s_{1}, v, c_{3}\right\}$ induces a claw. Then $v$ must be adjacent to $s_{2}$, for otherwise, $\left\{s_{2}, s_{1}, c_{1}, v\right\}$ induces a co-claw. Vertex $v$ is non-adjacent to $c_{2}$, for otherwise, $B$ and $v$ induce a 3 -sun, and we are done. Now we know $v \in X$. Similarly, if $v$ is adjacent to $c_{2}$, the $v \in Y$. If $X$ or $Y$ contains two vertices, then it is easy to see $G$ contains a claw, or co-claw. So, $G$ has at most seven vertices and is an induced subgraph of the graph $F_{2}$.

Let $\mathcal{F}_{1}$ (respectively, $\mathcal{F}_{2}$ ) be the class of graphs $G$ such that $G$ or $\bar{G}$ contains an antenna (respectively, bull) and is an induced subgraph of $F_{1}$ (respectively $F_{2}$ ).

Theorem 16. Let $G$ be a (claw, co-claw)-free graph. Then one of the following holds.

(i) $G$ or $\bar{G}$ contain no triangle.

(ii) $G$ or $\bar{G}$ is the net.

(iii) $G$ or $\bar{G}$ belong to $\mathcal{F}_{1}$.

(iv) $G$ or $\bar{G}$ belong to $\mathcal{F}_{2}$.

Proof. Let $G$ be a (claw, co-claw)-free graph. We may assume $G$ contains a triangle $T$ and a co-triangle $C$, for otherwise, (i) holds, and we are done. The sets $T$ and $C$ cannot be vertex-disjoint, for otherwise, (ii) holds by Theorems 12 and 13, and we are done. So, $T$ and $C$ must intersect at one vertex. It is easy to verify that $T \cup C$ induces a bull. We may assume $G$ contains no antenna, for otherwise, by Theorem 14, (iii) holds and we are done. By Theorem 15, $G$ belongs to $\mathcal{F}_{2}$, and so (iv) holds, and we are done. 
Corollary 17. If $G$ is a (claw, co-claw)-free graph that is not isomorphic to the net, then $G$ is e-positive.

Proof. This follows directly from the Theorem 16 and the facts that all graphs in $\mathcal{F}_{1}$ and $\mathcal{F}_{2}$ were verified by computer program to be $e$-positive, (claw, triangle)-free graphs and co-triangle-free graphs are $e$-positive, and the complement of the net is $e$-positive.

The following theorem is one of our main results:

Theorem 18. If $G$ is a co-claw-free unit interval graph, then $G$ is e-positive.

Proof. The only (claw, co-claw)-free graph that is not $e$-positive is the net and the net is not a unit interval graph (since it contains an astroidal triple). Then it follows from Corollary 17 that co-claw-free unit interval graphs are $e$-positive.

Corollary 19. If $G$ is a unit interval graph and the complement of $G$ is a unit interval graph, then $G$ is e-positive.

Proof. This follows directly from the previous theorem and the fact that if the complement of $G$ is a unit interval graph, then $G$ is co-claw-free.

In fact, the class of graphs stated in Theorem 18 can be shown to be $e$-positive by other means, i.e. this theorem does not provide any new examples of $e$-positive graphs. The following proof is due to Timothy Y. Chow [3]:

Proof. (Alternative proof of Theorem 18) If the graph is triangle-free, then since it is a unit interval graph, it must be a disjoint union of paths which, by [19] are $e$-positive. So assume $G$ contains a triangle. Since $G$ is a unit interval graph, assume $G$ is represented as a set of unit intervals with vertices arranged left to right and that there is a triangle formed by three consecutive vertices $a, b$, and $c$. Moreover, since all unit interval graphs with at most five vertices are known to be $e$-positive, we may assume $G$ has at least 6 vertices. This means there are two vertices we will call $d$ and $e$ either to the right of $c$ or to the left of $a$. Without loss of generality, assume they are to the right of $c$. But then all vertices to the right of $c$, and, in particular $d$ and $e$, must be adjacent to $c$ and to each other (since $G$ is co-claw-free) so these vertices all form a clique. By a similar argument, all vertices to the left of $d$ form a clique. Since $G$ is covered by two cliques, its complement cannot contain a triangle, and by Theorem 4, property 4, we know that complements of triangle-free graphs are $e$-positive.

\section{Strongly $e$-positive graphs}

As discussed in Section 1, the property of a graph being e-positive is not hereditary. This gives motivation to seek out which graphs have this special property. We coin the term strongly e-positive graphs for graphs with this property.

Definition 20. A graph $G$ is strongly e-positive if for all induced subgraphs $H$ of $G, H$ is $e$-positive. 
Note that the classes of claw-free co-comparability graphs, unit interval graphs, and (claw, co-diamond)-free graphs are all subclasses of (claw, net)-free graphs. See Figure 1. We conjecture that this class of graphs is exactly the class of strongly e-positive graphs.

Conjecture 21. A graph is strongly e-positive if and only if it is (claw, net)-free.

Clearly if a graph is strongly e-positive, then it is (claw, net)-free since both the claw and net are not $e$-positive. However, proving the other direction seems to be quite challenging. All (claw, net)-free graphs up to and including 9 vertices were verified by computer to be $e$-positive. This provides strong evidence in support of the conjecture.

The contrapositive of part of this conjecture is:

Conjecture 22. If $G$ is not $e$-positive, then $G$ contains an induced claw or an induced net.

Note a kinship between strongly e-positive and the nice property of Stanley [20] where a graph $G$ is nice if whenever there is a stable partition of $G$ of type $\lambda$ (i.e. a partition into stable sets of size $\left.\lambda_{1}, \lambda_{2}, \ldots\right)$ and whenever $\mu \leqslant \lambda$ in dominance order, there exists a stable partition of type $\mu$. Then Proposition 1.6 of [20] states that a graph $G$ and all its induced subgraphs are nice if and only if $G$ is claw-free.

We introduce a further conjecture that, in conjunction with a result from [12], would show that (claw, bull)-free graphs are e-positive. The work of [12] shows that every connected (claw, bull)-free graph is either the generalized graph of a path or cycle, or the complement of a triangle-free graph. As we know from Theorem 4, the complement of a triangle-free graph is e-positive, so the only remaining type of (claw, bull)-free graph to consider are those that take the form of the generalized graph of a path or cycle. Again, by Theorem 4 we know paths and cycles are $e$-positive. Creating the generalized graphs of paths or cycles is akin to adding what are called twin vertices (defined below). Conjecture 23 proposes that this twinning preserves $e$-positivity.

Two vertices $x$ and $y$ are twins (also called true twins) if they are adjacent and any vertex $z$ is adjacent to both $x$ and $y$ or non adjacent to both $x$ and $y$. Let $G$ be a graph, define $G^{\prime}$ to be the graph obtained from $G$ by adding a vertex $x$ that is a twin with some vertex $y$ in $G$. Then the following conjecture would be implied by Conjecture 22:

Conjecture 23. If $G$ is $e$-positive, then $G^{\prime}$ is $e$-positive.

\section{Acknowledgements}

The authors thank Timothy Y. Chow for permission to include the alternative proof of Theorem 18. This work was supported by the Canadian Tri-Council Research Support Fund. The authors A.M.H. and C.T.H. were each supported by individual NSERC Discovery Grants. This research was enabled in part by support provided by Compute Ontario (computeontario.ca) and Compute Canada (www. computecanada.ca). This work was done by author O.D.M. in partial fulfillment of the course requirements for CP493 and CP494: Directed Research Project I and II in the Department of Physics and Computer Science at Wilfrid Laurier University. 


\section{References}

[1] S. Cho, J. Huh, On e-positivity and e-unimodality of chromatic quasisymmetric functions, arXiv:1711.07152, 20 November 2017.

[2] S. Cho, S. van Willigenburg, Chromatic classical symmetric functions, J. Comb. 9 (2018), 401-409.

[3] T.Y. Chow, personal communication, 2018.

[4] S. Dahlberg, A. Foley, S. van Willigenburg, Resolving Stanley's e-positivity of clawcontractible-free graphs, arXiv:1703.05770, 16 March 2017.

[5] A. Foley, J. Kazdan, L. Kröll, S. Alberga, M. Martínez, O. Oleksii and A. Tenenbaum. Spiders and their Kin $\left(K_{n}\right)$, arXiv:1812.03476, 11 December 2018.

[6] F. Gardi, The Roberts characterization of proper and unit interval graphs, Discrete Mathematics, 307 (2007), 2906-2908.

[7] D. D. Gebhard, B. E. Sagan, A chromatic symmetric function in noncommuting variables, Journal of Algebraic Combinatorics 13 (2001), 227-255.

[8] M. Guay-Paquet, A modular relation for the chromatic symmetric functions of $(3+1)$ free posets, arXiv:1306.2400, 11 June 2013.

[9] M. Harada, M. Precup, The cohomology of abelian Hessenberg varieties and the Stanley-Stembridge conjecture, arXiv:1709.06736.

[10] A.M. Hamel, C. T. Hoàng, J.E. Tuero, Chromatic symmetric functions and $H$-free graphs, Graphs and Combinatorics, 35 (2019), 815-825.

[11] H. Hempel, D. Kratsch, On claw-free asteroidal triple-free graphs, Discrete Applied Mathematics 121 (2002), 155-180.

[12] S.G. Hermosillo de la Maza, Y. Jing, M. Masjoody, On the structure of (claw, bull)free graphs, arXiv:1901.00043, December 31, 2018.

[13] C.T. Hoàng, B. Reed, On the co- $P_{3}$-structure of perfect graphs, SIAM J. Disc. Math. 18 (2005), 571-576.

[14] T. Klocks, D. Kratsch, H. Müller, Approximating the bandwidth for asteroidal triplefree graphs, Journal of Algorithms 32 (1999), 41-57.

[15] C. Lekkerkerker, J. Boland, Representation of a finite graph by a set of intervals on the real line, Fundamenta Mathematicae, 51 (1962), 45-64.

[16] I.G. Macdonald, Symmetric Functions and Hall Polynomials, 2nd. Ed. Oxford: Oxford Univerity Press, 1995.

[17] J. Shareshian, M. Wachs, Chromatic quasisymmetric functions, Adv. in Math. 295 (2016), 497-551.

[18] R.P. Stanley, Enumerative Combinatorics, Vol 2, Cambridge: Cambridge University Press, 1999.

[19] R.P. Stanley, A symmetric function generalization of the chromatic polynomial of a graph, Adv. Math. 111 (1995), 166-194. 
[20] R.P. Stanley, Graph colorings and related symmetric functions: ideas and applications: a description of results, interesting applications, and notable open problems, Discrete Math. 193 (1998), 267-286.

[21] R. Stanley, J. R. Stembridge, On immanants of Jacobi Trudi matrices and permutations with restricted positions, J. Combin. Theory Ser. A 62 (1993), 261-279.

[22] S. Tsujie, The chromatic symmetric functions of trivially perfect graphs and cographs, Graphs and Combinatorics, 34 (2018), 1037-1048. 